\title{
Remarks on generalized scalar-tensor models of dark energy
}

\author{
M. Alimohammadi* and H. Behnamian \\ Department of Physics, University of Tehran, \\ North Karegar Avenue, Tehran, Iran.
}

\begin{abstract}
The generalized scalar-tensor models with Lagrangian $F(\phi, R)-U(\phi)(\nabla \phi)^{2}$ are considered. It is shown that the phantom-divide-line crossing and the deceleration to acceleration transition generally occurr in these models. Two specific examples, the coupled quintessence model and the BransDicke model are considered. For the first example, it is shown that for the models with $\xi>3 / 16$, the $\omega=-1$ transition exists. This is verified numerically for some special cases. For the Brans-Dicke model, it is shown that the transition does not occur, a result which can be verified by using the exact solution of this model. Finally the contribution of quantum effects on these phenomena is investigated. It is shown that for some special cases where the $\omega=-1$ transition is classically forbidden, the quantum effects can induce transition. The $\xi=1 / 6$ of coupled quintessence model is an example of this. The quantum effects are described via the account of conformal anomaly.
\end{abstract}

\section{Introduction}

Nowadays based on various observational data, it has been verified that our universe is now in an accelerating phase [1]. The source of this accelerated expansion, which is known as dark energy, is believed to compose nearly $70 \%$ of the present universe. The precise nature of dark energy is not yet known but all of the models describing the dark energy have a common property: they produce the repulsive force, or in other words, they have the negative pressure. Introducing the equation of state parameter by $\omega=p / \rho$, where $p$ is the pressure and $\rho$ is the energy density, it must satisfy $\omega<-1 / 3$, in Friedmann-RobertsonWalker (FRW) background metric, to ensure the accelerated expansion.

One of the interesting features of dark energy is the dynamical behavior of equation of state parameter $\omega$. This is because some astrophysical data slightly favor an evolving dark energy and show a recent $\omega=-1$, the so-called phantomdivide-line, crossing [2]. To describe the behavior of $\omega$, many dynamical dark energy models have been introduced. The simplest of these models are the single component scalar models, including the quintessence model, which consists of a normal scalar field [3], and the phantom model, which is a scalar field theory with unusual negative kinetic energy [4]. None of these models can describe the

*alimohmd@ut.ac.ir 
$\omega=-1$ crossing. In quintessence model $\omega$ is always $\omega>-1$, and in phantom model $\omega$ always satisfies $\omega<-1$. A possible way to overcome this problem is to consider the quintom model [5], the model consists of one quintessence and one phantom fields. It can be shown that the transition from $\omega>-1$ to $\omega<-1$ always occurs in the quintom model with slowly-varying potential [6]. The interaction of single-component scalar field with background (dark) matter can also induce this transition [7].

An alternative candidate of dark energy are the models known as modified gravities, in which it is postulated that the gravity is being nowadays modified by some extra terms which grow when the curvature decreases. The $f(R)$ gravity, whose action is a general function $f(R)$ of the Ricci scalar $R$, is the most famous modified gravity theory [8]. The $f(G)$ gravity and its generalization $F(R, G)$ gravity, where $G$ being the Gauss-Bonnet invariant, are also introduced [9]. Several aspects of these models, including their behavior under $\omega=-1$ transition, have been studied recently. It has been shown that the phantom-divide-line crossing and the deceleration to acceleration transition generally occur in $F(R, G)$ models. The contribution of quantum effects on these phenomenon has been also obtained [10].

An important class of modified gravity theories is the scalar-tensor theories. In these models, there exists a scalar field $\phi$, besides the usual space-time metric $g_{\mu \nu}$, to describe the gravitational interaction. The earliest type of these models is the Jordan-Brans-Dicke theory with the following action, in the so-called Jordan frame, [11]

$$
S=\frac{1}{2} \int \mathrm{d}^{4} x \sqrt{-g}\left[\phi R-\frac{\mathrm{w}}{\phi}(\nabla \phi)^{2}-V(\phi)\right]+S_{m} .
$$

Here we use the units in which $\hbar=c=8 \pi G=1$. ' $\mathrm{w}$ ' is a dimensionless parameter and $S_{m}$ is the action of dustlike matter. The scalar field $\phi$ plays the role of the inverse of the gravitational coupling, which is constant in general relativity but in Jordan-Brans-Dicke theory is variable. For the case where $V(\phi)=0$, the model is called the Brans-Dicke theory.

The generalization of the Jordan-Brans-Dicke theory is the scalar-tensor theory which is described, in the Jordan frame, by the action [12]

$$
S=\frac{1}{2} \int \mathrm{d}^{4} x \sqrt{-g}\left[F(\phi) R-U(\phi)(\nabla \phi)^{2}-V(\phi)\right]+S_{m} .
$$

Several aspects of scalar-tensor models have been studied, including their behavior as a dynamical system [13], the gravitational wave and inflation in this framework [14], and their perturbative aspects $[15,16]$. Two review articles on this subject are [17].

The most general action in the Jordan frame, based on the scalar-tensor theory, is the following:

$$
S=\frac{1}{2} \int \mathrm{d}^{4} x \sqrt{-g}\left[F(\phi, R)-U(\phi)(\nabla \phi)^{2}\right]+S_{m},
$$

which is called the generalized scalar-tensor (ST) theories [16,17]. The scalar theories, i.e. quintessence and phantom models, $f(R)$ gravity models, JordanBrans-Dicke theory (11), and the scalar-tensor models (2) are special examples of action (3). It is must be noted that the terminology is not fixed in scalar-tensor 
theories. The term "generalized scalar-tensor models" has been used for less general actions than eq.(3), even for action (2) with $F(\phi)=\phi$.

The present paper is devoted to the study of generalized ST theories. We want to study the conditions under which the $\omega=-1$ crossing occurs in generalized scalar-tensor theories. The condition of transition between deceleration and acceleration phases is also studied. We show that, through some specific examples, the phantom-divide-line crossing can not occur for some models. It is shown that by considering the quantum contributions, the situation becomes different. In fact, some of the forbidden transitions become allowed, i.e. the $\omega=-1$ transitions, for these cases, are quantum induced. The same phenomenon has been seen for quintessence and phantom theories [18]. The quantum effects are described via the account of conformal anomaly, reminding about anomaly-driven inflation [19]. The contribution of the conformal anomaly in energy conditions and Big Rip of phantom models has been discussed in [20].

The scheme of the paper is as follows. In section 2, the Friedmann equations of generalized ST theories for FRW space-time are obtained. By perturbative solving of these equations around the $\omega=-1$ and $\ddot{a}=0$ transition points in sections 3 and 4 , respectively, the conditions of occurrence of these crossings are obtained. In section 5 , we consider the explicit example of coupled quintessence model in which there exists a free parameter $\xi$. It is shown that the $\omega=-1$ transition occurs if $\xi>3 / 16$. It is seen that the numerical solving of Friedmann equations verifies our perturbative results. In section 6 , the Brans-Dicke model is considered as a second example. Our method shows no transition for this model, a result which can be verified by exact solution of this model. Finally in section 7 , we introduce the quantum contributions (comes from conformal anomaly) to this problem, and show that it can induce the $\omega=-1$ transition for special cases which are classically forbidden. For example for $\xi=1 / 6$ case of the coupled quintessence model, in which the transition is classically forbidden, the quantum phenomena induce the $\omega=-1$ transition.

\section{The generalized scalar-tensor models}

Consider the generalized scalar-tensor gravity with action (3). Varying this action with respect to the metric $g_{\mu \nu}$ results in [17]

$$
\begin{aligned}
& \left(R_{\mu \nu}-\nabla_{\mu} \nabla_{\nu}+g_{\mu \nu} \square\right) F_{R}(\phi, R)-\frac{1}{2} g_{\mu \nu}[F(\phi, R) \\
& \left.-U(\phi)(\nabla \phi)^{2}\right]-U(\phi) \nabla_{\mu} \phi \nabla_{\nu} \phi=T_{\mu \nu} .
\end{aligned}
$$

In this equation, $T_{\mu \nu}$ is the energy-momentum tensor of matter field

$$
T_{\mu \nu}=\frac{2}{\sqrt{-g}} \frac{\delta S_{m}}{\delta g_{\mu \nu}}
$$

and

$$
F_{R}(\phi, R)=\frac{\partial F(\phi, R)}{\partial R} \quad, \quad F_{\phi}(\phi, R)=\frac{\partial F(\phi, R)}{\partial \phi} .
$$

Varying the action with respect to the scalar field $\phi$, results in

$$
F_{\phi}(\phi, R)+2 U(\phi) \square \phi+2 \nabla^{\mu} \phi \nabla_{\mu} U(\phi)-\frac{d U(\phi)}{d \phi}(\nabla \phi)^{2}=0 .
$$


For the case where $U(\phi)=\eta=$ constant, eq.(17) reduces to the corresponding equation of motion in [17].

A spatially flat FRW space-time in comoving coordinates $(t, x, y, z)$ is defined through

$$
\mathrm{d} s^{2}=-\mathrm{d} t^{2}+a^{2}(t)\left(\mathrm{d} x^{2}+\mathrm{d} y^{2}+\mathrm{d} z^{2}\right),
$$

where $a(t)$ is the scale factor. For this metric, the $(t, t)$ component of the evolution equation (4) for homogenous scalar field $\phi(t)$ becomes:

$$
\begin{aligned}
& 3 H^{2} F_{R}(\phi, R)=\rho_{m}+\frac{1}{2} U(\phi) \dot{\phi}^{2}-3 H F_{R R}(\phi, R) \dot{R} \\
& -3 H F_{R \phi}(\phi, R) \dot{\phi}-\frac{1}{2} F(\phi, R)+\frac{1}{2} R F_{R}(\phi, R) .
\end{aligned}
$$

$H(t)=\dot{a}(t) / a(t)$ is the Hubble parameter and $\rho_{m}$ is the matter energy density with the evolution equation

$$
\dot{\rho}_{m}+3 H\left(\rho_{m}+p_{m}\right)=0 .
$$

$R$ is expressed in terms of $H$ as following

$$
R=6\left(\dot{H}+2 H^{2}\right) .
$$

The $(x, x)$ component of eq.(4), when eq.(9) is subtracted from it, becomes

$$
\begin{gathered}
-2 \dot{H} F_{R}(\phi, R)+H F_{R R}(\phi, R) \dot{R}+H F_{R \phi}(\phi, R) \dot{\phi}-F_{R R R}(\phi, R) \dot{R}^{2}-2 F_{R R \phi}(\phi, R) \dot{R} \dot{\phi} \\
-F_{R R}(\phi, R) \ddot{R}-F_{R \phi \phi}(\phi, R) \dot{\phi}^{2}-F_{R \phi}(\phi, R) \ddot{\phi}-U(\phi) \dot{\phi}^{2}=\gamma_{m} \rho_{m} .
\end{gathered}
$$

In above equation, $\gamma_{m}$ is defined by $\gamma_{m}=1+\omega_{m}$, where $\omega_{m}=p_{m} / \rho_{m}$, and the subscripts denote differentiations, e.g.

$$
F_{R R \phi}(\phi, R)=\frac{\partial^{3} F(\phi, R)}{\partial^{2} R \partial \phi} .
$$

Finally the scalar field equation of motion (7) in FRW metric becomes:

$$
F_{\phi}(\phi, R)-2 U(\phi)(\ddot{\phi}+3 H \dot{\phi})-\dot{U}(\phi) \dot{\phi}=0 .
$$

In above equation $\dot{U}=(\mathrm{d} U / \mathrm{d} \phi) \dot{\phi}$. Equations (9), (10), (12) and (14) are the Friedmann equations of generalized scalar-tensor models. Note that these four equations are not independent, in fact, taking the time derivative of eq.(9), adding it eq.(12), multiplied by $3 H$, and using eq.(14), result in eq.(10). So there are three independent Friedmann equations. In the case of ordinary scalar-tensor models where $F(\phi, R)=R F(\phi)-V(\phi)$, the resulting Friedmann equations lead to the corresponding ones in scalar-tensor theories.

\section{The phantom-divide-line crossing}

For the ordinary dark energy models and in the context of Einstein gravity, the equation of state parameter $\omega=p / \rho$ is obtained via $\omega=-1-2 \dot{H} / 3 H^{2}$. For 
other theories, including the usual and generalized ST theories, the effective equation of state parameter $\omega_{\text {eff }}$ is also defined through $[9,16]$

$$
\omega_{\text {eff }}=-1-\frac{2}{3} \frac{\dot{H}}{H^{2}} .
$$

So $\omega_{\text {eff }}$ crosses the phantom-divide-line, i.e. $\omega_{\text {eff }}=-1$, if $H(t)$ has a relative extremum at some time $t=t_{0}$. Restricting ourselves to $t-t_{0} \ll h_{0}^{-1}$, where $h_{0}=H\left(t_{0}\right)$ and $h_{0}^{-1}$ is of order of the age of our universe, the Hubble parameter can be expanded as

$$
H(t)=h_{0}+h_{1}\left(t-t_{0}\right)^{\alpha}+h_{2}\left(t-t_{0}\right)^{\alpha+1}+O\left(\left(t-t_{0}\right)^{\alpha+2}\right),
$$

in which $\alpha \geq 2$ is the order of the first nonvanishing derivative of $H(t)$ at $t=t_{0}$, and $h_{1}=(1 / \alpha !) H^{(\alpha)}\left(t_{0}\right) . H^{(n)}\left(t_{0}\right)$ is $n$th derivative of $H(t)$ at $t=t_{0}$. The transition occurs from $\omega>-1$ to $\omega<-1$ regions when $\alpha$ is an even positive integer and $h_{1}>0$. For $h_{1}<0$, and even integer $\alpha$, the system goes from $\omega<-1$ to $\omega>-1$ at $t=t_{0}$. We now examine whether there is any solution for Friedmann eqs.(9), (10), and (14), if $H(t)$ is given by (16). If for an even positive integer $\alpha$, we can find a nonzero value for $h_{1}$, then the possibility of $\omega=-1$ transition of generalized ST theories is proved.

We first consider the Friedmann equation (9):

$$
H^{2} F_{R}(\phi, R) \equiv \beta(t),
$$

in which

$$
\begin{gathered}
\beta(t)=\frac{1}{3}\left[\rho_{m}+\frac{1}{2} U(\phi) \dot{\phi}^{2}-3 H F_{R R}(\phi, R) \dot{R}\right. \\
\left.-3 H F_{R \phi}(\phi, R) \dot{\phi}-\frac{1}{2} F(\phi, R)+\frac{1}{2} R F_{R}(\phi, R)\right] .
\end{gathered}
$$

Expanding both sides of eq.(17) around $t_{0} \equiv 0$, one finds, up to order $t^{2}$,:

$$
\begin{gathered}
h_{0}^{2} F_{R}(0)=\beta(0), \\
h_{0}^{2}\left(F_{R R} \dot{R}+F_{R \phi} \dot{\phi}\right)_{t=0}=\dot{\beta}(0),
\end{gathered}
$$

and

$$
\begin{aligned}
\frac{1}{2} h_{0}^{2}\left(F_{R R R} \dot{R}^{2}\right. & \left.+2 F_{R R \phi} \dot{R} \dot{\phi}+F_{R R} \ddot{R}+F_{R \phi \phi} \dot{\phi}^{2}+F_{R \phi} \ddot{\phi}\right)_{t=0} \\
& +2 h_{0} h_{1} F_{R}(0) \delta_{\alpha, 2}=\frac{1}{2} \ddot{\beta}(0) .
\end{aligned}
$$

The second Friedmann equation (10), at zero order, leads to

$$
\dot{\rho}_{m}(0)+3 h_{0} \gamma_{m} \rho_{m}(0)=0
$$

and the last equation of motion (14), up to order $t$, results in:

$$
F_{\phi}(0)-2 U(0)\left(\ddot{\phi}+3 h_{0} \dot{\phi}\right)_{t=0}-U_{\phi}(0) \dot{\phi}^{2}(0)=0,
$$

and

$$
\begin{gathered}
F_{\phi \phi}(0) \dot{\phi}(0)-U_{\phi}(0)\left(4 \dot{\phi} \ddot{\phi}+6 h_{0} \dot{\phi}^{2}\right)_{t=0}-2 U(0)\left(\dddot{\phi}+3 h_{0} \ddot{\phi}\right)_{t=0} \\
-U_{\phi \phi}(0) \dot{\phi}^{3}(0)+12 h_{1} F_{R \phi}(0) \delta_{\alpha, 2}=0 .
\end{gathered}
$$


Using eqs.(11), (22) and (23), the eqs.(19) and (20) lead to:

$$
\begin{aligned}
& 3 h_{0}^{2} F_{R}(0)+\rho_{m}(0)+\frac{1}{2} U(0) \dot{\phi}^{2}(0)-\frac{1}{2} F(0) \\
& -3 h_{0} F_{R \phi}(0) \dot{\phi}(0)-36 h_{0} h_{1} F_{R R}(0) \delta_{\alpha, 2}=0
\end{aligned}
$$

and

$$
\begin{gathered}
6\left[3 h_{0}\left(h_{2}+h_{0} h_{1}\right) F_{R R}+12 h_{0} h_{1}^{2} F_{R R R}+2 h_{0} h_{1} F_{R R \phi} \dot{\phi}\right]_{t=0} \delta_{\alpha, 2}+18 h_{0} h_{1} F_{R R}(0) \delta_{\alpha, 3} \\
+\frac{1}{2}\left[\gamma_{m} \rho_{m}-h_{0} F_{R \phi} \dot{\phi}+U(\phi) \dot{\phi}^{2}+F_{R \phi \phi} \dot{\phi}^{2}+F_{R \phi} \ddot{\phi}\right]_{t=o} h_{0}=0
\end{gathered}
$$

respectively. Note that at $t=0, R=R(0)=12 h_{0}^{2}$.

Let us first consider the case $\alpha=2$. The eqs.(22), (25) and (26) then result in $h_{0}, h_{1}$ and $h_{2}$, respectively. $h_{0}$ and $h_{1}$ are

$$
h_{0}=-\frac{\dot{\rho}_{m}(0)}{3 \gamma_{m} \rho_{m}(0)},
$$

and

$$
h_{1}=\frac{1}{36 h_{0} F_{R R}(0)}\left[3 h_{0}^{2} F_{R}+\rho_{m}+\frac{1}{2} U(\phi) \dot{\phi}^{2}-\frac{1}{2} F-3 h_{0} F_{R \phi} \dot{\phi}\right]_{t=0} .
$$

So the parameter $h_{1}$ is, in general, different from zero, which depends on initial values $\rho_{m}(0), \dot{\rho}_{m}(0), \phi(0)$, and $\dot{\phi}(0)$, can be positive or negative. This proves that, in general, the generalized ST theories can explain the phantom-divideline crossing. The remaining two equations (23) and (24) determine the values of $\ddot{\phi}(0)$ and $\dddot{\phi}(0)$ in terms of the four mentioned initial values. The higher order terms of the expansions specify the other coefficients $h_{3}, h_{4}, \ldots$ For example eq.(21) gives the parameter $h_{3}$.

It is clear that when the Lagrangian $F(\phi, R)$ is linear in $R$, as is the case for scalar-tensor theories with action (2), then eq.(28) is not the solution (note that in this case, the last term of eq.(25) vanishes). For these cases, it can be shown that eq.(21) specifics $h_{1}$ as follows:

$$
\begin{gathered}
h_{1}=\frac{1}{4 F_{R}(0)}\left[3 \rho_{m} \gamma_{m}^{2} h_{0}+6 h_{0} U(\phi) \dot{\phi}^{2}-F_{\phi} \dot{\phi}+F_{R \phi}\left(h_{0} \ddot{\phi}-\dddot{\phi}\right)\right. \\
\left.+F_{R \phi \phi}\left(h_{0} \dot{\phi}^{2}-3 \dot{\phi} \ddot{\phi}\right)-F_{R \phi \phi \phi} \dot{\phi}^{3}\right]_{t=0} .
\end{gathered}
$$

For the next choice $\alpha=3$, it is clear from eq.(25) that it does not lead to an expression for $h_{1}$, unlike the case $\alpha=2$ in which eq.(25) leads to (28), but instead, this equation leads to an extra relation between the initial values $\rho_{m}(0)$, $\dot{\rho}_{m}(0), \phi(0)$ and $\dot{\phi}(0)$. For $\alpha=3$, eq.(26) determines $h_{1}$. So the $\alpha=3$ solution is also possible whenever there exists a special relation between the four initial values. The situation is worse for $\alpha \geq 4$, since in these cases eq.(26) also leads to another relation between the initial values. So except for these fine-tuned initial values, the only acceptable solution is $\alpha=2$, which always permits the $\omega=-1$ transition. 


\section{The deceleration to acceleration transition}

To study the transition from $\ddot{a}<0$ to $\ddot{a}>0$, we first note that

$$
\frac{\ddot{a}}{a}=\dot{H}+H^{2}
$$

So at time $t_{0}^{\prime} \equiv 0$ where $\ddot{a}=0$, one has $\dot{H}=-H^{2}$. Expanding $H(t)$ around $t_{0}^{\prime}=0$

$$
H(t)=H_{0}+H_{1} t+H_{2} t^{2}+\cdots
$$

then $\dot{H}=-\left.H^{2}\right|_{t=0}$ results in

$$
H_{1}=-H_{0}^{2}
$$

Similar to the previous section, one can seek the solutions for eqs.(9), (10) and (14), when $H(t)$ is given by (31), with condition (32). In this case, the resulting relations for $H_{0}$ and $H_{2}$ are

$$
H_{0}=-\frac{\dot{\rho}_{m}(0)}{3 \gamma_{m} \rho_{m}(0)}
$$

and

$$
H_{2}=\frac{1}{36 H_{0} F_{R R}(0)}\left[\rho_{m}+\frac{1}{2} U(\phi) \dot{\phi}^{2}+72 H_{0}^{4} F_{R R}-3 H_{0} F_{R \phi} \dot{\phi}-\frac{1}{2} F\right]_{t=0},
$$

respectively. The other coefficients of expansion (31) can be also found consistently, which proves the existence of $\ddot{a}=0$ crossing in generalized ST theories. For the cases where $F(\phi, R)$ is linear in $R, H_{2}$ in eq. (34) is not more the solution and another expression, like eq.(29) for $\omega=-1$ crossing, can be found.

\section{The coupled quintessence model}

As an explicit example, we consider the coupled quintessence model introduced by following action: [17]

$$
S=\frac{1}{2} \int \mathrm{d}^{4} x \sqrt{-g}\left[\left(1-\xi \phi^{2}\right) R-(\nabla \phi)^{2}\right]+S_{m}
$$

in which we do not consider the potential $V(\phi)$. At $\xi=0$, eq. (35) becomes the ordinary quintessence model, and for $\xi \neq 0$, the scalar field $\phi$ coupled nonminimally to the gravity. The reason for considering the parameter $\xi$, first introduced in [21], has several answers which have been discussed in [17]. Perhaps the main reason to include a $\xi \neq 0$ term is that it is introduced by the first loop corrections and is required by normalizability of the theory [22]. If one considers a classical theory with $\xi=0$, renormalization shifts it to one with $\xi \neq 0$. We will return to the quantum corrections in section 7 .

In this section, we study the conditions under which the $\omega=-1$ transition occurs in $V(\phi)=0$ coupled quintessence model. For $\alpha=2$, our zero-order relations are eqs.(22), (23) and (25). Since here we will not consider the matter 
field, for simplicity, the eq.(22), which is the Friedmann equation (10) at zero order, is not suitable. Instead we consider eq.(12) and our three independent Friedmann equations become eqs.(91), (12) and (14), where for action (35), result in:

$$
\begin{gathered}
6 H^{2}\left(1-\xi \phi^{2}\right)-\dot{\phi}^{2}-12 H \xi \phi \dot{\phi}-2 \rho=0, \\
-2 \dot{H}\left(1-\xi \phi^{2}\right)-2 H \xi \phi \dot{\phi}+2 \xi \phi \ddot{\phi}+(2 \xi-1) \dot{\phi}^{2}=\rho+p,
\end{gathered}
$$

and

$$
\ddot{\phi}+3 H \dot{\phi}+\xi \phi\left(6 \dot{H}+12 H^{2}\right)=0,
$$

respectively. At zero order, these equations lead to:

$$
\begin{gathered}
\dot{\phi}_{0}^{2}+12 \xi h_{0} \phi_{0} \dot{\phi}_{0}-6 h_{0}^{2}\left(1-\xi \phi_{0}^{2}\right)=0, \\
-2 \xi h_{0} \phi_{0} \dot{\phi}_{0}+2 \xi \phi_{0} \ddot{\phi}_{0}+(2 \xi-1) \dot{\phi}_{0}^{2}=0, \\
\ddot{\phi}_{0}+3 h_{0} \dot{\phi}_{0}+12 \xi h_{0}^{2} \phi_{0}=0,
\end{gathered}
$$

respectively. Here we assume that $S_{m}=0$. The first equation specifies $\dot{\phi}_{0}$ in terms of $\phi_{0}$, and by eliminating $\ddot{\phi}_{0}$ between eqs. (40) and (41), one more expression for $\dot{\phi}_{0}$, in terms of $\phi_{0}$, is obtained. These relations are

$$
\dot{\phi}_{0}=-h_{0}\left[6 \xi \phi_{0} \pm \sqrt{36 \xi^{2} \phi_{0}^{2}+6\left(1-\xi \phi_{0}^{2}\right)}\right],
$$

and

$$
\dot{\phi}_{0}=-\frac{h_{0}}{1-2 \xi}\left[4 \xi \phi_{0} \pm \xi \phi_{0} \sqrt{8(6 \xi-1)}\right]
$$

respectively. Note that eq. (43), which comes from eqs.(37) and (38), holds only at the $\omega=-1$ transition point. This is because by setting $\dot{H}=0$, which is the signature of $\omega=-1$ transition, eqs.(37) and (38) lead to (40) and (41) and then (43) is found. This is not the case for eq.(42) which holds at any instant of time.

The reality constraint of the scalar field $\phi$, restricts $\xi$ in eq. (43) by

$$
\xi \geq \frac{1}{6}
$$

Setting equal the eqs. (42) and (43), results in

$$
2 \xi \phi_{0} \sqrt{6 \xi-1}[\sqrt{6 \xi-1} \pm \sqrt{2}]= \pm(1-2 \xi) \sqrt{36 \xi^{2} \phi_{0}^{2}+6\left(1-\xi \phi_{0}^{2}\right)} .
$$

Note that for $\xi \geq 1 / 2$, the left-hand-side of eq.(45) is positive, so in the righthand-side, only the minus sign must be used for $\xi \geq 1 / 2$. Solving $\phi_{0}$ from eq.(45), one finds

$\phi_{0}^{( \pm)^{2}}=\frac{3(1-2 \xi)^{2}}{2 \xi^{2}}\left[(6 \xi-1)(6 \xi+1 \pm 2 \sqrt{12 \xi-2})-9(1-2 \xi)^{2}+\frac{3(1-2 \xi)^{2}}{2 \xi}\right]^{-1}$.

It can be easily seen that $\phi_{0}^{(-)^{2}}$ is always negative for all $\xi \geq 1 / 6$, so there exists no real solution for $\phi_{0}^{(-)}$. For $\phi_{0}^{(+)^{2}}$, it can be seen that $\phi_{0}^{(+)^{2}}<0$ for 
$1 / 6 \leq \xi \leq 3 / 16$ and $\phi_{0}^{(+)^{2}}>0$ for $\xi>3 / 16$. So the real value of the scalar field $\phi$ at transition time $t=0$, is $\phi_{0}^{(+)}$from eq.(46), and it exists if

$$
\xi>\frac{3}{16}
$$

In other words, the coupled quintessence model can cross the $\omega=-1$ line only when $\xi$ satisfies eq. (47). This leads us to consider the eq.42) and the righthand-side of eq. (45) with "+" and "-" sign, for $3 / 16<\xi<1 / 2$ and $\xi \geq 1 / 2$, respectively 1 .

To determine the crossing behavior of this models, we must calculate the parameter $h_{1}$ from eq.(29), or instead, from perturbative calculation of eq.(12), or (37). To do so, it is better to rewrite the differential eqs.(36)-(38) in terms of red-shift parameter $z$, defined through

$$
1+z=\frac{a_{*}}{a},
$$

instead of the time variable $t$. In eq.(48), $a_{*}$ is the scale factor of the universe in the present time. Using

$$
\begin{aligned}
& \frac{\mathrm{d}}{\mathrm{d} t}=-H(1+z) \frac{\mathrm{d}}{\mathrm{d} z}, \\
& \frac{\mathrm{d}^{2}}{\mathrm{~d} t^{2}}=H^{2}(1+z)^{2} \frac{\mathrm{d}^{2}}{\mathrm{~d} z^{2}}+H(1+z)^{2} \frac{\mathrm{d} H}{\mathrm{~d} z} \frac{\mathrm{d}}{\mathrm{d} z}+H^{2}(1+z) \frac{\mathrm{d}}{\mathrm{d} z},
\end{aligned}
$$

the first Friedmann equation (36) results in $\eta(z)=\mathrm{d} \phi / \mathrm{d} z$ in terms of $\phi(z)$, and eqs. (37) and (38) specify $\mathrm{d} H / \mathrm{d} z$ and $\mathrm{d} \eta / \mathrm{d} z$. The resulting equations are:

$$
\begin{gathered}
\eta(z)=\frac{\mathrm{d} \phi(z)}{\mathrm{d} z} \\
(1+z) \eta(z)=6 \xi \phi(z) \pm \sqrt{36 \xi^{2} \phi^{2}(z)+6\left(1-\xi \phi^{2}(z)\right)} \\
\frac{\mathrm{d} H(z)}{\mathrm{d} z}=\frac{24 \xi^{2} \phi^{2}(z)+(1-2 \xi)(1+z)^{2} \eta^{2}(z)-8 \xi(1+z) \eta(z) \phi(z)}{2(1+z)\left[1+\left(6 \xi^{2}-\xi\right) \phi^{2}(z)\right]} H(z) \\
\frac{\mathrm{d} \eta(z)}{\mathrm{d} z}=\frac{1}{2(1+z)\left[1+\left(6 \xi^{2}-\xi\right) \phi^{2}(z)\right]} \times\left\{24 \xi^{2} \phi^{3}(z)+(2 \xi-1)(1+z)^{3} \eta^{3}(z)\right. \\
\left.+4(1+z)\left[1-\left(\xi+12 \xi^{2}\right) \phi^{2}(z)\right] \eta(z)+2(1+z)^{2}\left(7 \xi-6 \xi^{2}\right) \eta^{2}(z) \phi(z)-24 \xi \phi(z)\right\}
\end{gathered}
$$

As explained after eq.(47), for $3 / 16<\xi<1 / 2$, eq.(51) must be used with " +" sign and for $\xi \geq 1 / 2$, the " - " sign is accepted.

Expanding $H(z)$ around the transition point $z_{0}$, one finds

$$
H(z)=h_{0}+h_{1}^{\prime}\left(z-z_{0}\right)^{2}+h_{2}^{\prime}\left(z-z_{0}\right)^{3}+\cdots .
$$

\footnotetext{
${ }^{1}$ Since only the plus sign of eq. (46) , or eq. (43) and the LHS of eq. (45), leads to acceptable solution for $\phi_{0}$, we must consider the eq. (43) and the LHS of eq. with " + "sign. Under this condition, the LHS of eq. (45) becomes positive for $3 / 16<\xi<1 / 2$, therefore the " + " sign must be chosen in the RHS of this equation and therefore in eq.422). For $\xi \geq 1 / 2$, the accepted sign in the RHS of eq.4(45) and in eq. (42) is " - ", as pointed out after eq.455.
} 
in which we choose $\alpha=2$. Note that $\omega=-1-(2 / 3) \dot{H} / H^{2}=-1+\frac{2(1+z)}{3 H} \mathrm{~d} H / \mathrm{d} z$, so at transition point $z_{0}$, one has $\mathrm{d} H /\left.\mathrm{d} z\right|_{z=z_{0}}=0$. It is clear from eq.(152) that $\mathrm{d} H / \mathrm{d} z=A(z) H(z)$, so $A\left(z_{0}\right)=0$ from which $\mathrm{d}^{2} H /\left.\mathrm{d} z^{2}\right|_{z=z_{0}}=H(z) \mathrm{d} A /\left.\mathrm{d} z\right|_{z=z_{0}}$. In this way, eqs.(50)-(53) finally result in $h_{1}^{\prime}=(1 / 2) \mathrm{d}^{2} H /\left.\mathrm{d} z^{2}\right|_{z=z_{0}}$ as follows

$$
\begin{aligned}
& h_{1}^{\prime}=\frac{h_{0}}{8\left(1+z_{0}\right)^{2}\left[1+\left(6 \xi^{2}-\xi\right) \phi_{0}^{2}\right]^{2}} \times\left\{96\left(1+z_{0}\right)\left[\left(6 \xi^{4}+\xi^{3}+\xi^{2}\right) \phi_{0}^{2}+2 \xi^{2}-\xi\right] \phi_{0} \eta_{0}\right. \\
& +4\left(1+z_{0}\right)^{2}\left[\left(36 \xi^{3}-36 \xi^{2}-3 \xi\right) \phi_{0}^{2}-10 \xi+3\right] \eta_{0}^{2}+12\left(1+z_{0}\right)^{3}\left(4 \xi^{3}-6 \xi^{2}+3 \xi\right) \phi_{0} \eta_{0}^{3} \\
& \left.-2\left(1+z_{0}\right)^{4}\left(4 \xi^{2}-4 \xi+1\right) \eta_{0}^{4}+192 \xi^{2} \phi_{0}^{2}-192 \xi^{3} \eta_{0}^{4}\right\} .
\end{aligned}
$$

In this equation, $\phi_{0}=\phi\left(z_{0}\right)$ and $\eta_{0}=\eta\left(z_{0}\right)$.

At the first step, we solve numerically the differential equations (50), (52) and (53) for the specific example $\xi=1$. By choosing the initial values $\phi(z=0)=1 / 2$ and $H(z=0)=72$ and calculating $\eta(z=0)$ from eq. (51) with " - "sign, because $\xi>1 / 2$, one finds $H(z), \phi(z), \eta(z)$ and $\omega(z)=-1+\frac{2(1+z)}{3 H} \mathrm{~d} H / \mathrm{d} z$ in Figs.(1)-(4).

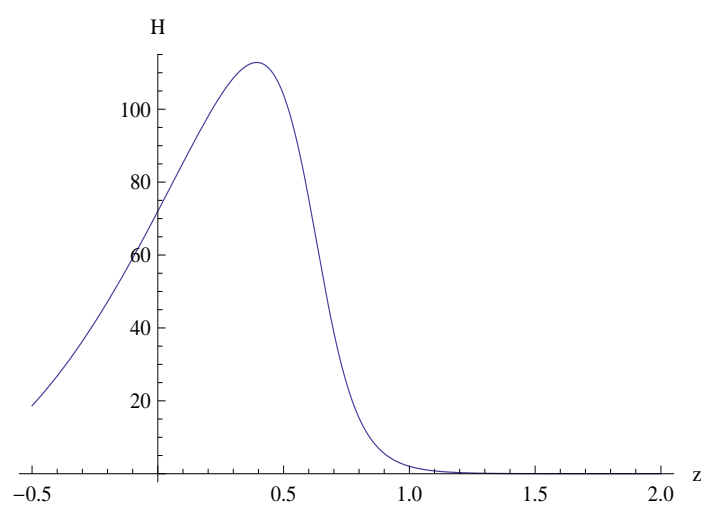

Figure 1: The plot of $H(z)$ of coupled quintessence model with $\xi=1$.

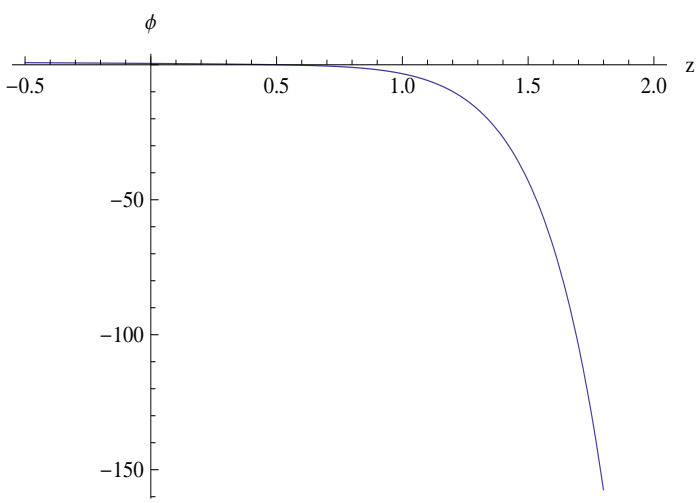

Figure 2: The plot of $\phi(z)$ of coupled quintessence model with $\xi=1$. 


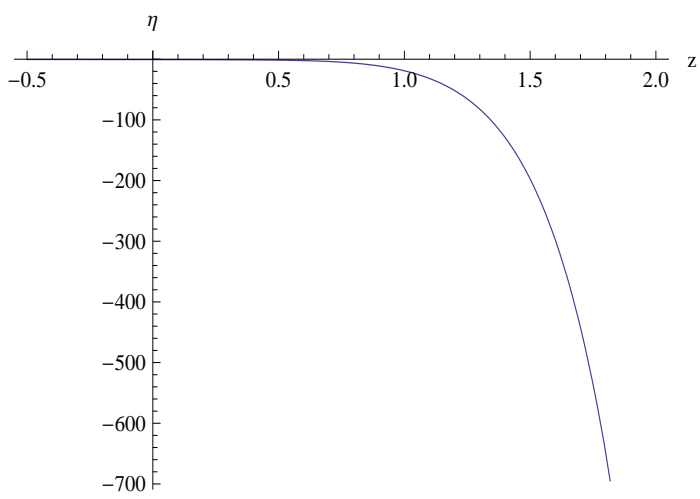

Figure 3: The plot of $\eta(z)=\mathrm{d} \phi(z) / \mathrm{d} z$ of coupled quintessence model with $\xi=1$.

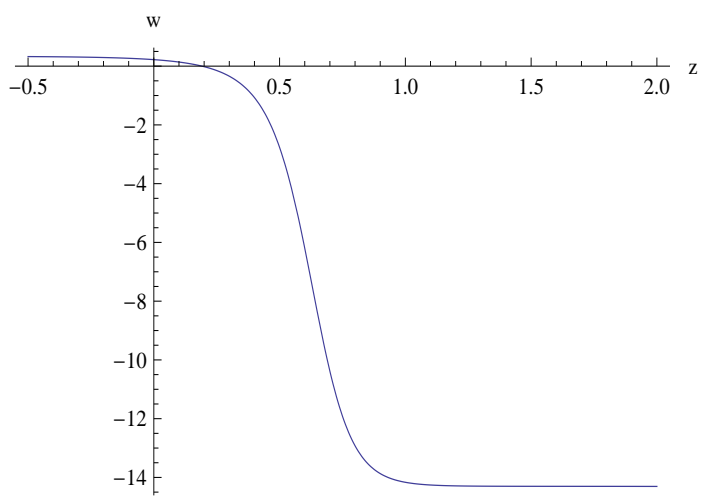

Figure 4: The plot of $\omega(z)$ of coupled quintessence model with $\xi=1$. It crosses $\omega=-1$ at $z_{0}=0.393$.

As it is clear from Fig.(4), $\omega(z)$ crosses the $\omega=-1$ line, from $\omega>-1$ region to $\omega<-1$ region, at $z_{0}=0.393$. At this point, $H\left(z_{0}\right)=112.83, \phi\left(z_{0}\right)=0.16$, and $\eta\left(z_{0}\right)=-1.18$.

Our perturbative results verify this crossing. For $\xi=1, \phi_{0}^{(+)}$from eq. (46) becomes $\phi_{0}^{(+)}=0.16$, which is in coincidence with numerical calculations. Choosing $z_{0}=0.393$ and $h_{0}=112.83$, eq.(51) results in $\eta\left(z_{0}\right)=-1.18$, and $h_{1}^{\prime}$ from eq.(55) becomes $h_{1}^{\prime}=-618.9$. Therefore, up to lowest order, we have:

$$
\begin{aligned}
& \omega(z)=-1+\frac{2(1+z)}{3 H(z)} \frac{\mathrm{d} H}{\mathrm{~d} z}=-1+\frac{4 h_{1}^{\prime}}{3 h_{0}}(1+z)\left(z-z_{0}\right)+\cdots \\
& =-1-7.31(1+z)(z-0.393)+\cdots
\end{aligned}
$$

Fig.(5) shows the relation (56), which has the similar behavior, near the transition point $z=z_{0}$, as the Fig.(4). Note that in both approaches, the number of needed initial values are the same. In numerical calculation, we use two initial values $\phi(z=0)$ and $H(z=0)$, and in perturbative calculation, two parameters $z_{0}$ and $h_{0}=H\left(z=z_{0}\right)$ are chosen. 


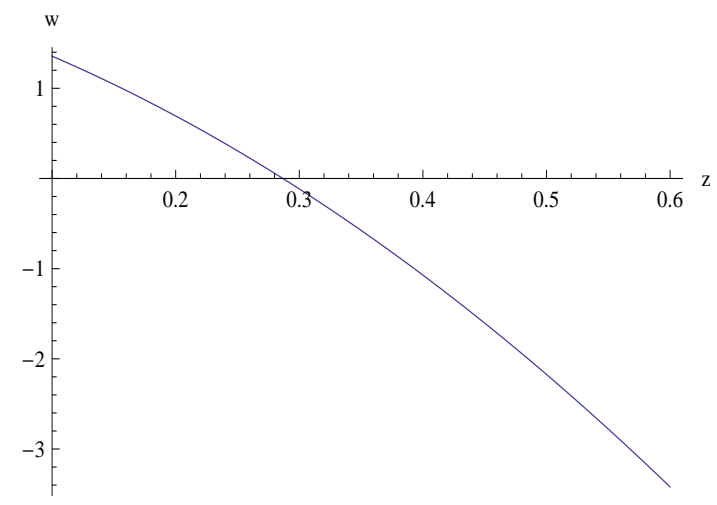

Figure 5: The perturbative variation of $\omega(z)$ of coupled quintessence model with $\xi=1$.

\section{The Brans-Dicke model}

As a second example, we consider the Jordan-Brans-Dicke model with $V(\phi)=0$ and $S_{m}=0$,

$$
S=\frac{1}{2} \int \mathrm{d}^{4} x \sqrt{-g}\left[\phi R-\frac{\mathrm{w}}{\phi}(\nabla \phi)^{2}\right] .
$$

Comparing (57) with (37), gives $F(\phi)=R \phi$ and $U(\phi)=\mathrm{w} / \phi$. In this way, eqs.(23)-(26), with $\alpha=2$, and (29) become:

$$
\begin{gathered}
12 h_{0}^{2}+\mathrm{w} A^{2}-2 \mathrm{w}\left(3 h_{0} A+B\right)=0, \\
-C-A^{3}+2 A B+3 h_{0} A^{2}-3 h_{0} B+\frac{6}{\mathrm{w}} h_{1}=0, \\
-\mathrm{w} A^{2}+6 h_{0} A+6 h_{0}^{2}-2 \frac{\rho_{0}}{\phi_{0}}=0, \\
B+\mathrm{w} A^{2}-h_{0} A+\frac{\rho_{0}+p_{0}}{\phi_{0}}=0,
\end{gathered}
$$

and

$$
h_{1}=\frac{1}{4}\left[-C+h_{0} B+6 h_{0} \mathrm{w} A^{2}-12 h_{0}^{2} A+\frac{3 \rho_{0} \gamma^{2} h_{0}}{\phi_{0}}\right],
$$

respectively. In above equations, the parameters $A, B$, and $C$ are

$$
A=\left(\frac{\dot{\phi}}{\phi}\right)_{0} \quad, \quad B=\left(\frac{\ddot{\phi}}{\phi}\right)_{0} \quad, \quad C=\left(\frac{\dddot{\phi}}{\phi}\right)_{0},
$$

and $R(0)=12 h_{0}^{2}$ has been used. Setting $\rho_{0}=p_{0}=0$ in eqs.(58)-(62), to ensure $S_{m}=0$, eq.(60) then gives $A$ as follows:

$$
A=\frac{3 h_{0}}{\mathrm{w}}\left[1 \pm \sqrt{1+\frac{2 \mathrm{w}}{3}}\right] .
$$

Reality of $A$ demands

$$
\mathrm{w}>-\frac{3}{2},
$$


where for the reasons which will be discussed later, we do not consider the $\mathrm{w}=-3 / 2$ case.

The parameter $B$ can be found from either eqs.(58) or (61). Using eq.(60), they yield

$$
B=-\left(5 h_{0} A+6 h_{0}^{2}\right)=\frac{9}{\mathrm{w}} h_{0}^{2}+3\left(\frac{1}{\mathrm{w}}-1\right) h_{0} A .
$$

Putting (64) into eq.(66), the parameter 'w' is fixed as

$$
\mathrm{w}=-\frac{4}{3} \text {. }
$$

In other words, the Brans-Dicke theory has $\omega=-1$ only when $\mathrm{w}=-4 / 3$. Putting (67) back into eq.(64), with plus sign, and eq.(66), results in

$$
A=-3 h_{0} \quad, \quad B=9 h_{0}^{2} .
$$

The parameter $h_{1}$ can be found by either eqs.(59) and (62), where using (67) and (68), it gives the result

$$
h_{1}=-\frac{2}{9}\left(C+27 h_{0}^{3}\right)=-\frac{1}{4}\left(C+27 h_{0}^{3}\right) .
$$

The above equality gives $C$ :

$$
C=-27 h_{0}^{3},
$$

from which

$$
h_{1}=0 .
$$

So it seems that there is no $\omega=-1$ crossing phenomenon in Brans-Dicke model. In fact, calculating the other coefficients of expansion (16) results in $h_{i}(i \geq 1)=$ 0 .

To justify our result, let us exactly calculate the equation of state parameter $\omega$ of the Brans-Dicke model. We first write eqs.(91), (12) and (14) for this model, which results in

$$
\begin{gathered}
3 H^{2} \phi=\frac{1}{2} \frac{\mathrm{w}}{\phi} \dot{\phi}^{2}-3 H \dot{\phi}, \\
-2 \dot{H} \phi+H \dot{\phi}-\ddot{\phi}-\frac{\mathrm{w}}{\phi} \dot{\phi}^{2}=0,
\end{gathered}
$$

and

$$
6\left(\dot{H}+2 H^{2}\right)-2 \frac{\mathrm{w}}{\phi}(\ddot{\phi}+3 H \dot{\phi})+\frac{\mathrm{w}}{\phi^{2}} \dot{\phi}^{2}=0,
$$

respectively. Using the red-shift parameter $z$, the above equations can be used to obtain the following relations in $z$-space:

$$
\begin{gathered}
6=\mathrm{w}(1+z)^{2}\left(\frac{\eta(z)}{\phi(z)}\right)^{2}+6(1+z) \frac{\eta(z)}{\phi(z)} \\
\frac{\mathrm{d} H(z)}{\mathrm{d} z}=\frac{H(z)}{(1+z)(6+4 \mathrm{w})}\left[\mathrm{w}(1+2 \mathrm{w})(1+\mathrm{z})^{2}\left(\frac{\eta(\mathrm{z})}{\phi(\mathrm{z})}\right)^{2}+8 \mathrm{w}(1+\mathrm{z}) \frac{\eta(\mathrm{z})}{\phi(\mathrm{z})}+12\right]
\end{gathered}
$$

${ }^{2}$ Note that for the minus sign of eq. (64), the solution of eq.(66) is $\mathrm{w}=-3 / 2$, and for the plus sign it is $\mathrm{w}=-3 / 2$ and $\mathrm{w}=-4 / 3$. Because of the physical condition [65, $\mathrm{w}=-4 / 3$ is the only acceptable solution and therefore we only consider the plus sign of eq. (64). 
and

$$
\begin{aligned}
& \frac{\mathrm{d} \eta(z)}{\mathrm{d} z}=\frac{\phi(z)}{(1+z)(6+4 \mathrm{w})}\left[-\mathrm{w}(1+2 \mathrm{w})(1+z)^{2} \frac{\eta^{3}(z)}{\phi^{3}(z)}\right. \\
& \left.-12 \mathrm{w}(1+z) \frac{\eta^{2}(z)}{\phi^{2}(z)}+8(\mathrm{w}-3) \frac{\eta(z)}{\phi(z)}+\frac{24}{1+z}\right]
\end{aligned}
$$

in which $\eta(z)=\mathrm{d} \phi(z) / \mathrm{d} z$. Because of the denominator of eqs.(76) and (77), we take $\mathrm{w} \neq-3 / 2$ for these equations. We will consider the $\mathrm{w}=-3 / 2$ case separately. Solving eq.(75) for $\eta(z) / \phi(z)$, results in

$$
\frac{\eta(z)}{\phi(z)}=\frac{1}{\mathrm{w}(1+z)}[-3 \pm \sqrt{9+6 \mathrm{w}}],
$$

in which we assume $\mathrm{w} \neq 0$ and also the condition (65). Putting eq.(78) into eq.(76), gives

$$
\frac{\mathrm{d} H(z)}{\mathrm{d} z}=\frac{g_{\mp}(\mathrm{w})}{1+z} H(z),
$$

where

$$
g_{\mp}(\mathrm{w})=\frac{1}{\mathrm{w}}[3(\mathrm{w}+1) \mp \sqrt{3(2 \mathrm{w}+3)}] .
$$

In this way the exact solution of the Brans-Dicke model is found as follows:

$$
H(z)=H\left(z_{0}\right)(1+z)^{g_{\mp}(\mathrm{w})} .
$$

The equation of state parameter $\omega$ can be found as

$$
\omega(z)=-1+\frac{2}{3} \frac{(1+z)}{H(z)} \frac{\mathrm{d} H}{\mathrm{~d} z}=-1+\frac{2}{3} g_{\mp}(\mathrm{w}),
$$

which is constant in time. So it is natural that it does not cross the phantomdivide-line, and $\omega(z)=-1$ occurs when $g_{\mp}(\mathrm{w})=0 . \quad g_{-}(\mathrm{w})=0$ results in $\mathrm{w}=0$ which is not acceptable, and $g_{+}(\mathrm{w})=0$ results in $\mathrm{w}=-4 / 3$ which is in accordance with the perturbative result obtained in eq.(67).

It is interesting to note that our exact solution (81) coincides with O'Hanlon and Tupper solution: [23]

$$
\begin{aligned}
& a(t)=a_{0}\left(\frac{t}{t_{0}}\right)^{q_{ \pm}}, \\
& \phi(t)=\phi_{0}\left(\frac{t}{t_{0}}\right)^{s_{ \pm}},
\end{aligned}
$$

with

$$
\begin{aligned}
q_{ \pm} & =\frac{\mathrm{w}}{3(\mathrm{w}+1) \mp \sqrt{3(2 \mathrm{w}+3)}}, \\
s_{ \pm} & =\frac{1 \mp \sqrt{3(2 \mathrm{w}+3)}}{3 \mathrm{w}+4} .
\end{aligned}
$$

In $t$-space, 833) results in

$$
H(t)=\frac{q_{ \pm}}{t} \Rightarrow \omega=-1-\frac{2}{3} \frac{\dot{H}}{H^{2}}=-1+\frac{2}{3 q_{ \pm}},
$$


which by noting that $g_{\mp}=1 / q_{ \pm}$(see eqs. (80) and (850), the $\omega$ in (86) becomes the same as one in eq.(82).

The only remaining case is $\mathrm{w}=-3 / 2$. To see the behavior of the Brans-Dicke model at $\mathrm{w}=-3 / 2$, we consider the Friedmann equations (72)-(74). From the first equation (72), one finds

$$
\frac{1}{H} \frac{\dot{\phi}}{\phi}=\left.\frac{3 \pm \sqrt{9+6 \mathrm{w}}}{\mathrm{w}}\right|_{\mathrm{w}=-\frac{3}{2}}=-2,
$$

from which

$$
\begin{aligned}
& \dot{\phi}=-2 H \phi, \\
& \ddot{\phi}=-2 \dot{H} \phi+4 H^{2} \phi .
\end{aligned}
$$

Putting these equations back into the second and third Friedmann equations (73) and (74), which are not independent at $\mathrm{w}=-3 / 2$, it can be easily seen that these equations trivially hold for arbitrary $H(t)$ function. Therefore we exclude $\mathrm{w}=-3 / 2$ case in which all the $H(t)$ functions are solutions of the Friedmann equations.

\section{The quantum corrections}

As it was discussed earlier, the classical Lagrangian

$$
\mathcal{L}=\frac{1}{2}\left(-\xi R \phi^{2}-(\nabla \phi)^{2}\right)
$$

can induce interesting quantum effects. The renormalizability requirements enforces $\xi$ in the above action to be $\xi=1 / 6$. In $\xi=1 / 6$, the model has several other interesting properties, such as the conformal invariance, holding the Einstein equivalence principle, etc. $[17,22]$.

Calculating the effective action of this model at one-loop level, results in some extra terms in the trace of the energy-momentum tensor, which is traceless classically. For this reason, this effect is called the trace/conformal anomaly. These extra terms are $[19,22]$.

$$
T=b\left(F+\frac{2}{3} \square R\right)+b^{\prime} G+b^{\prime \prime} \square R,
$$

where $T$ denotes the trace of the energy-momentum tensor, $F$ is the square of the $4 \mathrm{~d}$ Weyl tensor and $G$ is the Gauss-Bonnet invariant

$$
\begin{aligned}
& F=\frac{1}{3} R^{2}-2 R_{\mu \nu} R^{\mu \nu}+R_{\mu \nu \alpha \beta} R^{\mu \nu \alpha \beta}, \\
& G=R^{2}-4 R_{\mu \nu} R^{\mu \nu}+R_{\mu \nu \alpha \beta} R^{\mu \nu \alpha \beta} .
\end{aligned}
$$

For $N$ scalars, $N_{1 / 2}$ spinors, $N_{1}$ vector fields, $N_{2}(=0$ or 1$)$ gravitons and $N_{H D}$ higher derivative conformal scalars, $b, b^{\prime}$ and $b^{\prime \prime}$ are given by

$$
\begin{aligned}
& b=\frac{N+6 N_{1 / 2}+12 N_{1}+611 N_{2}-8 N_{H D}}{120(4 \pi)^{2}}, \\
& b^{\prime}=-\frac{N+11 N_{1 / 2}+62 N_{1}+1411 N_{2}-28 N_{H D}}{360(4 \pi)^{2}}, \quad b^{\prime \prime}=0 .
\end{aligned}
$$


Using eq.(90) for the case of FRW metric, it can be shown that the contribution of conformal anomaly to energy density and pressure are [24]

$$
\begin{aligned}
& \rho_{A}=-\frac{1}{a^{4}}\left\{b^{\prime}\left(6 a^{4} H^{4}+12 a^{2} H^{2}\right)\right. \\
& +\left(\frac{2}{3} b+b^{\prime \prime}\right)\left[a^{4}\left(-6 H \ddot{H}-18 H^{2} \dot{H}+3 \dot{H}^{2}\right)+6 a^{2} H^{2}\right] \\
& \left.-2 b+6 b^{\prime}-3 b^{\prime \prime}\right\}
\end{aligned}
$$

and

$$
\begin{aligned}
& p_{A}=b^{\prime}\left[6 H^{4}+8 H^{2} \dot{H}+\frac{1}{a^{2}}\left(4 H^{2}+8 \dot{H}\right)\right] \\
& +\left(\frac{2}{3} b+b^{\prime \prime}\right)\left[-2 \dddot{H}-12 H \ddot{H}-18 H^{2} \dot{H}-9 \dot{H}^{2}+\frac{1}{a^{2}}\left(2 H^{2}+4 \dot{H}\right)\right] \\
& -\frac{-2 b+6 b^{\prime}-3 b^{\prime \prime}}{3 a^{4}} .
\end{aligned}
$$

The subscript " $A$ " stands for "anomaly". To consider the quantum effects in gravitational phenomena, one can add the above $\rho_{A}$ and $p_{A}$ to the Friedmann equations. In this way the contribution of quantum effects on $\omega=-1$ crossing can be studied.

\subsection{The coupled-quintessence model}

We first study how much the adding of the quantum terms $\rho_{A}$ and $p_{A}$ can change the $\omega=-1$ crossing of the coupled quintessence model discussed in section 5. As this model is a theory with one scalar field, one has $N=1$ and $N_{1 / 2}=N_{1}=N_{2}=N_{H D}=0$. Eq.(92) then results in

$$
b=-3 b^{\prime}=\frac{1}{120(4 \pi)^{2}} .
$$

The Friedmann equations of coupled quintessence model are eqs.(36)-(38), in which we put $\rho_{A}$ and $p_{A}$ as the source terms. At zero-order, eqs.93) and (94), using (95), are

$$
\rho_{A}(0)=-b^{\prime}\left(8 h_{0}^{4}+24 h_{0} h_{1}+\frac{12}{a_{0}^{4}}\right),
$$

and

$$
p_{A}(0)=b^{\prime}\left(6 h_{0}^{4}+48 h_{0} h_{1}-\frac{4}{a_{0}^{4}}+24 h_{2}\right),
$$

respectively. Therefore, instead of eqs.(39) and (40), now we have:

$$
\dot{\phi}_{0}^{2}+12 \xi h_{0} \phi_{0} \dot{\phi}_{0}-6 h_{0}^{2}\left(1-\xi \phi_{0}^{2}\right)-2 b^{\prime}\left(8 h_{0}^{4}+24 h_{0} h_{1}+\frac{12}{a_{0}^{4}}\right)=0,
$$

and

$$
-2 \xi h_{0} \phi_{0} \dot{\phi}_{0}+2 \xi \phi_{0} \ddot{\phi}_{0}+(2 \xi-1) \dot{\phi}_{0}^{2}=b^{\prime}\left(-2 h_{0}^{4}+24 h_{0} h_{1}-\frac{16}{a_{0}^{4}}+24 h_{2}\right),
$$

respectively. Eq.(41) is not changed. As it is clear from the above equations, the situation is very different from classical relations (39)-(41). Here we do 
not have the relations like eqs. (42) and (43) which describe $\dot{\phi}_{0}$ in terms of $\phi_{0}$, and therefore the constraint like eq.(47) does not appear. In other words, the $\omega=-1$ crossing can happen for any $\xi$ values. In fact, eq.98) determines $h_{1}$ as follows:

$$
h_{1}=\frac{\dot{\phi}_{0}^{2}+12 \xi h_{0} \phi_{0} \dot{\phi}_{0}-6 h_{0}^{2}\left(1-\xi \phi_{0}^{2}\right)-8 b^{\prime}\left(2 h_{0}^{4}+3 / a_{0}^{4}\right)}{48 b^{\prime} h_{0}},
$$

which is generally different from zero for any $\xi$ values, including $\xi=1 / 6$. In this way we obtain an interesting result: The coupled quintessence model classically crosses the $\omega=-1$ line for $\xi>3 / 16$, but because of the quantum effects, the $\xi=1 / 6<3 / 16$ is also allowed. It is interesting to note that the quantum correction terms (the last two terms in the numerator of eq.(100) ) are much smaller than the classical terms, since

$$
h_{0}^{4} \sim \frac{1}{a_{0}^{4}} \ll h_{0}^{2} .
$$

\subsection{The Brans-Dicke model}

In this case, the quantum induced energy density and pressure (96) and (97) must be inserted into eqs.(58)-(62), in order to find the influence of quantum phenomena on $\omega=-1$ transition of Brans-Dicke model. Eq. (60) then becomes

$$
-\mathrm{w} \frac{\dot{\phi}_{0}^{2}}{\phi_{0}}+6 h_{0} \dot{\phi}_{0}+6 h_{0}^{2} \phi_{0}+2 b^{\prime}\left(8 h_{0}^{4}+24 h_{0} h_{1}+\frac{12}{a_{0}^{4}}\right)=0 .
$$

In contrast to classical case in which eq. 60 , with $\rho_{0}=0$, specifies $(\dot{\phi} / \phi)_{0}$ in terms of "w" in eq. 64), from which $\mathrm{w}=-4 / 3$ becomes necessary for achieving $\omega=-1$, see eq.(67), and finally results in $h_{1}=0$ in eq.(71), here the eq.(60), in its new form (102), results in a nonzero $h_{1}$ :

$$
h_{1}=\frac{\mathrm{w} \dot{\phi}_{0}^{2} / \phi_{0}-6 h_{0} \dot{\phi}_{0}-6 h_{0}^{2} \phi_{0}-8 b^{\prime}\left(2 h_{0}^{4}+3 / a_{0}^{4}\right)}{48 b^{\prime} h_{0}} .
$$

This shows that the quantum effects can produce the non-zero $h_{1}$ which indicates the existence of $\omega=-1$ crossing.

There is a last point which must be noted. Comparing the Brans-Dicke Lagrangian (57) with Lagrangian (89), which $\rho_{A}$ and $p_{A}$ in eqs. (96) and (97) are derived from, may lead us to conclude that it is not reasonable to use $\rho_{A}$ and $p_{A}$ as the quantum corrections of (57). This is because these two Lagrangians are different, so the corrections come from (89) can not be used for (57). The answer is that if we apply the following change of field variable to eq. (57):

$$
\phi \rightarrow \frac{1}{4 \mathrm{w}} \phi^{2},
$$

the Brans-Dicke action then becomes

$$
S=\frac{1}{2} \int \mathrm{d}^{4} x \sqrt{-g}\left(\frac{1}{4 \mathrm{w}} R \phi^{2}-(\nabla \phi)^{2}\right) .
$$

By choosing $\xi=-1 / 4 \mathrm{w}$, eq.(105) becomes the same as the Lagrangian (89), so its quantum computations can be used for Brans-Dicke action. Restricting 
ourselves to $\xi=1 / 6$, results in $\mathrm{w}=-3 / 2$, which is not in the region of our interest. So in the physical region $\mathrm{w}>-3 / 2$, the quantum phenomena can not change the noncrossing behavior of equation of state parameter from $\omega=-1$ line.

\subsection{Deceleration to acceleration transition}

To study the influences of quantum effects on $\ddot{a}=0$ transition, the quantum terms $\rho_{A}$ and $p_{A}$ must be added to Friedmann equations (9), (10) and (14), and solved for the coefficients of the expansion $H(t)$ in eq. (31). Eq. (33) does not change, but the coefficient $H_{2}$ in eq.(34), for $b=-3 b^{\prime}$ case, is replaced by

$$
\begin{gathered}
H_{2}=\frac{1}{12 H_{0}\left(2 b^{\prime}+3 F_{R R}(0)\right)}\left[\rho_{m}+\frac{1}{2} U(\phi) \dot{\phi}^{2}+72 H_{0}^{4} F_{R R}-3 H_{0} F_{R \phi} \dot{\phi}\right. \\
\left.-\frac{1}{2} F+12 b^{\prime}\left(3 H_{0}^{4}-\frac{1}{a_{0}^{4}}\right)\right]_{t=0} .
\end{gathered}
$$

In above equation, $a_{0}$ is the scale factor $a(t)$ at the transition time. It is seen that in $b^{\prime} \rightarrow 0$ limit, eq. (106) reduces to classical relation (34), as it is expected. For special cases coupled quintessence and Brans-Dicke models, $\mathrm{H}_{2}$ can be easily calculated from eq.(106).

\section{Conclusion}

In this paper, we consider the generalized scalar-tensor models as a class of modified gravity theories to describe two important cosmological transitions, the $\omega=-1$ and $\ddot{a}=0$ transitions. For both cases, we solve the Friedmann equations of generalized ST models by perturbative expansion of the Hubble parameter around the transition points. The expansion parameters are found consistently, which proves the existence of these solutions.

Two specific examples are studied in details. The first one is the coupled quintessence model, which the scalar field is coupled to gravity nonminimally. It is shown that for all $\xi>3 / 16$ cases, the model has a $\omega=-1$ transition, a fact which can be seen by numerical solving of the Friedmann equations for several $\xi$-values. The $\xi=1$ results are reported. The second example is the Brans-Dicke model, which our perturbative method shows no transition, a fact that is verified by solving exactly the Friedmann equations of this model. It is seen that the equation of state parameter of this model is constant, which naturally never cross the $\omega=-1$ line. Our investigation shows that "w" must satisfy $\mathrm{w}>-3 / 2$.

Finally we consider the quantum terms coming from the one-loop calculation of a scalar field, coupled non-minimally to gravity. It is shown that these quantum field theoretical terms can, in general, change the $\omega=-1$ and $\ddot{a}=0$ crossing behaviors of the models. For instance it is shown that the coupled quintessence model with $\xi=1 / 6$, which classically can not cross the $\omega=-1$ line, crosses this line because of quantum effects. For Brans-Dicke model, this phenomenon has no effect on transition of models with $\mathrm{w}>-3 / 2$.

Acknowledgement: This work was partially supported by the "center of excellence in structure of matter" of the Department of Physics of the University of Tehran, and also a research grant from the University of Tehran 


\section{References}

[1] A. G. Riess et al., [ Supernova Search Team Collaboration ], Astron. J. 116 (1998) 1009; S. Perlmutter et al., [ Supernova Cosmology Project Collaboration ], Astrophys. J. 517 (1999) 565.

[2] D. Huterer and A. Cooray, Phys. Rev. D 71 (2005) 023506; S. Nesserisa and L. Perivolaropoulos, Phys. Rev. D 72 (2005) 123519; U. Seljak, A. Slosar and P. McDonald, J. Cosmol. Astropart. Phys. 10 (2006) 014.

[3] A. R. Liddle, P. Parson and J. D. Barrow, Phys. Rev. D 50 (1994) 7222; R. R. Caldwell, R. Dave and P. J. Steinhardt, Phys. Rev. Lett. 80 (1998) 1582; P. J. E. Peebles and A. Vilenkin, Phys. Rev. D 59 (1999) 063505; M. Doran and J. Jaeckel, Phys. Rev. D 66 (2002) 043519; H. Ziaeepour, Phys. Rev. D 69 (2004) 063512; M. Garny, Phys. Rev. D 74 (2006) 043009; X. Zhang, Phys. Lett. B 648 (2007) 1; E. V. Linder, Gen. Relativ. Gravit. 40 (2008) 329; S. Y. Zhou, Phys. Lett. B 660 (2008) 7.

[4] R. R. Caldwell, Phys. Lett. B 545 (2002) 23; R. R. Caldwell, M. Kamionkowski and N. N. Weinberg, Phys. Rev. Lett. 91 (2003) 071301; J. M. Cline, S. Jeon and G. D. Moore, Phys. Rev. D 70 (2004) 043543; D. Samart and B. Gumjudpai, Phys. Rev. D 76 (2007) 043514; O. Hrycyna and M. Szydlowski, Phys. Lett. B 651 (2007) 8.

[5] B. Feng, X. -L. Wang and X. -M. Zhang, Phys. Lett. B 607 (2005) 35; R. Lazkov, G. Leon and I. Quiros, Phys. Lett. B 649 (2007) 103; M. Alimohammadi, Gen. Relativ. Gravit. 40 (2008) 107.

[6] H. Mohseni Sadjadi and M. Alimohammadi, Phys. Rev. D 74 (2006) 043506; M. Alimohammadi and H. Mohseni Sadjadi, Phys. Lett. B 648 (2007) 113.

[7] L. P. Chimento, A. S. Jakubi, D. Pavon and W. Zimdahl, Phys. Rev. D 67 (2003) 083513; H. Mohseni Sadjadi and M. Alimohammadi, Phys. Rev. D 74 (2006) 103007; H. Mohseni Sadjadi, arXiv:0904.1349 gr-qc].

[8] S. Nojiri and S. D. Odintsov, Phys. Rev. D 68 (2003) 123512; V. Faraoni, Phys. Rev. D 76 (2007) 127501; S. Caroll, V. Duvvuri, M. Trodden and M. Turner, Phys. Rev. D 70 (2004) 043528; J. Evans, L. Hall and P. Caillol, Phys. Rev. D 77 (2008) 083514.

[9] S. Nojiri and S. D. Odintsov, Phys. Lett. B 631 (2005) 1; S. Nojiri, S. D. Odintsov and O. G. Grobunova, J. Phys. A 39 (2006) 6627; G. Cognola, E. Elizalde, S. Nojiri, S. D. Odintsov and S. Zerbini, Phys. Rev. D 73 (2006) 084007.

[10] M. Alimohammadi and A. Ghalee, Phys. Rev. D 79 (2009) 063006.

[11] P. Jordan, Naturwiss 26 (1938) 417; M. Fierz, Helv. Phys. Acta 29 (1956) 128; C. H. Brans and R. H. Dicke, Phys. Rev. 124 (1961) 925. 
[12] P. G. Bergmann, Int. J. Theor. Phys. 1 (1968) 25; K. Nordtvedt, Astrophys. J. 161 (1970) 1059; R. V. Wagoner, Phys. Rev. D 1 (1970) 3209 .

[13] A. Billyard, A. Coley and J. Ibanez, Phys. Rev. D 59 (1998) 023507; E. Gunzig, V. Faraoni, A. Figueiredo, T. M. Rocha and L. Brenig, Class. Quant. Grav. 17 (2000) 1783; E. Gunzig, A. Saa, L. Brenig, V. Faraoni, T. M. Rocha Filho and A. Figueiredo, Phys. Rev. D 63 (2001) 067301; F. C. Carvalho and A. Saa, Phys. Rev. D 70 (2004) 087302; L. Jarv, P. Kuusk, and M. Saal, Phys. Rev. D 75 (2007) 023505; O. Hrycyna and M. Szydlowski, Phys. Rev. D 76 (2007) 123510.

[14] D. La and P. J. Steinhardt, Phys. Rev. Lett. 62 (1989) 376; C. M. Will "Theory and Experiment in Gravitational Physics", Cambridge University Press, Cambridge, England, 1993.

[15] B. Boisseau, G. Eposito-Farese, D. Polarski and A. A. Starobinsky, Phys. Rev. Lett. 85 (2000) 2236; F. Perrotta, S. Matarrese, M. Pietroni and C. Schmid, Phys. Rev. D 69 (2004) 084004.

[16] S. Tsujikawa, K. Uddin, S. Mizuno, R. Tavakol and J. Yokoyama, Phys. Rev. D 77 (2008) 103009.

[17] V. Faraoni, Ann. Phys. (NY) 317 (2005) 366; V. Faraoni, "Cosmology in Scalar-Tensor Gravity", Kluwer Academic Publishers, Netherlands, 2004 .

[18] M. Alimohammadi and L. Sadeghian, J. Cosmol. Astropart. Phys. 01 (2009) 035.

[19] A. Starobinsky, Phys. Lett. B 91 (1980) 99.

[20] S. Nojiri and S. D. Odintsov, Phys. Lett. B 562 (2003) 147; Phys. Lett. B 595 (2004) 1; Phys. Rev. D 70 (2004) 103522.

[21] N. A. Chernikov and E. A. Tagirov, Ann. Inst. H. Poincare A 9 (1968) 109; C. G. Callan, Jr., S. Coleman and R. Jackiw, Ann. Phys. (NY) 59 (1970) 42.

[22] N. D. Birrell and P. C. W. Davies "Quantum fields in curved space" Cambridge University Press, Cambridge, England, 1986.

[23] J. O'Hanlon and B. Tupper, Nuovo Cimento B 7 (1972) 305.

[24] S. Nojiri, S. D. Odintsov and S. Ogushi, Int. J. Mod. Phys. A 17 (2002) 4809; S. Nojiri and S. D. Odintsov, Int. J. Mod. Phys. A 16 (2001) 3273. 